\title{
Perimicrovillar membrane assembly: the fate of phospholipids synthesised by the midgut of Rhodnius prolixus
}

\author{
Paula Rêgo Bittencourt-Cunha, ${ }^{1,5}$, Livia Silva-Cardoso, ${ }^{1,5}$, Giselle Almeida de Oliveira ${ }^{1}$, \\ José Roberto da Silva ${ }^{2,5}$, Alan Barbosa da Silveira, ${ }^{1,5}$, George Eduardo Gabriel Kluck, ${ }^{1,5}$, \\ Michele Souza-Lima ${ }^{1,5}$, Katia Calp Gondim, ${ }^{1,5}$, Marilvia Dansa-Petretsky, ${ }^{3,5}$, Carlos Peres Silva ${ }^{4}$, \\ Hatisaburo Masuda ${ }^{1,5}$, Mario Alberto Cardoso da Silva Neto ${ }^{1,5}$, Georgia Correa Atella ${ }^{1,5} /+$ \\ 'Instituto de Bioquímica Médica, Centro de Ciências da Saúde, Universidade Federal do Rio de Janeiro, Rio de Janeiro, RJ, Brasil \\ ${ }^{2}$ Departamento de Bioquímica, Instituto de Química, Universidade Federal do Rio de Janeiro, Macaé, RJ, Brasil \\ ${ }^{3}$ Centro de Biociências e Biotecnologia, Universidade Estadual Norte Fluminense, Campos dos Goytacazes, RJ, Brasil \\ ${ }^{4}$ Departamento de Bioquímica, Instituto de Ciências Biológicas, Universidade Federal de Santa Catarina, Florianópolis, SC, Brasil \\ ${ }^{5}$ Instituto Nacional de Ciência e Tecnologia em Entomologia Molecular, Rio de Janeiro, RJ, Brasil
}

In this study, we describe the fate of fatty acids that are incorporated from the lumen by the posterior midgut epithelium of Rhodnius prolixus and the biosynthesis of lipids. We also demonstrate that neutral lipids (NL) are transferred to the haemolymphatic lipophorin $(L p)$ and that phospholipids remain in the tissue in which they are organised into perimicrovillar membranes (PMMs). ${ }^{3} \mathrm{H}$-palmitic acid added at the luminal side of isolated midguts of $\mathrm{R}$. prolixus females was readily absorbed and was used to synthesise phospholipids (80\%) and NL (20\%). The highest incorporation of ${ }^{3} \mathrm{H}$-palmitic acid was on the first day after a blood meal. The amounts of diacylglycerol (DG) and triacylglycerol synthesised by the tissue decreased in the presence of Lp in the incubation medium. The metabolic fates of ${ }^{3} H$-lipids synthesised by the posterior midgut were followed and it was observed that DG was the major lipid released to Lp particles. However, the majority of phospholipids were not transferred to Lp, but remained in the tissue. The phospholipids that were synthesised and accumulated in the posterior midgut were found to be associated with Rhodnius luminal contents as structural components of PMMs.

Key words: lipid metabolism - lipoprotein - lipid transport - phospholipids - midgut

The importance and the physiological role of the midgut in lipid metabolism have been studied by several authors in different insect species. It is widely recognised that the main region responsible for digestion in insects is the midgut, which contains cells that secrete digestive enzymes and are permeable to the products of digestion (Wigglesworth 1928, Nogueira et al. 1997, Terra et al. 2006, Mury et al. 2010). The midgut also participates in the absorption and processing of dietary lipids and the exportation of these molecules to the haemolymph. Triacylglycerol (TG) is a major component of the diet for some insects and is a major form of fatty acid (FA) storage (Beenakkers et al. 1985). In the midgut of Locusta migratoria, the products of TG hydrolysis are rapidly incorporated into phospholipids and neutral lipids (NL) (Weintraub \& Tietz 1973). In addition, the midguts of Pieris brassicae and Aeshna cyanea incorporate triolein and oleic acid from the diet and transfer them to the haemolymph, mainly in the form of diacylglycerol (DG) (Turunen 1975, Bauerfeind \& Komnick 1992, Grillo et al. 2007). Soon after the haematophagous bug Rhodnius

doi: 10.1590/0074-0276108042013016

Financial support: CNPq, IFS, FAPERJ, CAPES

+ Corresponding author: atella@bioqmed.ufrj.br

Received 12 November 2012

Accepted 10 April 2013 prolixus has a blood meal, phospholipids and DGs are released from the midgut to lipophorin (Lp) particles. However, on the 10th day after feeding, a dramatic shift in lipid metabolism occurs and the midgut becomes the main site of lipid incorporation (Atella et al. 1995, Coelho et al. 1997).

Most of the studies concerning lipid incorporation by the midgut have analysed dietary absorption; little work has been conducted with respect to lipid uptake from the haemolymph. Atella et al. (2000) demonstrated that when ${ }^{14} \mathrm{C}$-oleic acid was injected into the haemocoel of adult females 10 days after a blood meal, the radioactive oleic acid rapidly associated with Lp particles. Thirty minutes after the injection, the radioactivity was distributed among different organs; however, the midgut was the main site of radioactivity incorporation because this is where FAs are primarily used to synthesise phospholipids.

The hemipterans also differ from other insects because they lack a peritrophic membrane and their midgut cells are coated with an extracellular membrane, the perimicrovillar membrane (PMM) (Terra 1988). This structure was described and interpreted differently in morphological and enzymological studies performed by several authors (Marshall \& Cheung 1970, Reger 1971, Burgos \& Gutiérrez 1976, Lane \& Harrison 1979, Baerwald \& Delcarpio 1983, Billingsley \& Downe 1986, Ferreira et al. 1988, Silva et al. 1996). The PMM is a lipoprotein structure that extends from the base of the microvilli towards the lumen and forms a closed compart- 
ment known as the perimicrovillar space (Terra 1988). This space is located between the microvillar membrane and the PMM. Some roles of the PMM are analogous to peritrophic membrane functions, such as the compartmentalisation of the digestive process, the optimisation of amino acid absorption and the immobilisation of some enzymes. Additionally, the PMM physically and chemically protects the intestinal epithelium against the toxic products of blood digestion (Silva et al. 2007). Previous investigations have determined that the PMM of $R$. prolixus is also involved in the adherence of trypanosomatids to the intestine. These studies suggested that PMM molecules, such as lectins, carbohydrates and glycoproteins, are related to the competence of Trypanosoma cruzi to colonise the midgut of the vector successfully (Pereira \& Königk 1981, Isola et al. 1986, Mello et al. 1996, Ratcliffe et al. 1996, Bourguignon et al. 1998, Kollien et al. 1998, Gonzalez et al. 1999, Araújo et al. 2002, Alves et al. 2007).

Although some studies have investigated the possible functions of the PMM, research on the synthesis and lipid composition of the PMM is lacking. In this study, we describe the fates of the FAs that are incorporated into the posterior midgut of $R$. prolixus and the biosynthesis of lipids. We also demonstrate that NL are transferred to the haemolymphatic Lp and that phospholipids remain in the tissue, where they are organised into PMMs.

\section{MATERIALS AND METHODS}

Insects - Insects were taken from a colony of $R$. prolixus maintained at $28^{\circ} \mathrm{C}$ and $70-80 \%$ relative humidity. The experimental insects were adult mated females that fed on rabbit blood at three-week intervals.

Midgut labelling with a radioactive FA - The posterior midguts from adult females were isolated, washed and tied at both ends. ${ }^{3} \mathrm{H}$-palmitic acid $\left[0.05 \mu \mathrm{L}, 10^{6}\right.$ counts per minute (cpm); specific activity: $57 \mathrm{mCi} / \mathrm{mmol}$ ] (Perkin Elmer, Waltham, Massachusetts, USA) was injected into the luminal side of these posterior midguts using a PLI-100 microinjector (Harvard Apparatus, Holliston, Massachusetts, USA). In the experiments designed to study total lipid metabolism and the release of lipids to $\mathrm{Lp}$, the ${ }^{3} \mathrm{H}$-palmitic acid-injected midguts were incubated in 199 culture medium (Sigma, St. Louis, Missouri, USA) enriched with $10 \mu \mathrm{g} / \mu \mathrm{L}$ non-radioactive purified Lp. Unless otherwise stated, the incubations were performed at $28^{\circ} \mathrm{C}$. At the indicated times, the culture media or the midgut were removed and subjected to lipid extraction and the lipids were analysed by thin-layer chromatography (TLC).

Lipid analysis - The lipids extracted (Bligh \& Dyer 1959) from Lp, posterior midguts, luminal contents or PMMs were analysed by TLC, as described previously for phospholipids (Horwitz \& Perlman 1987) or NL (Kawooya \& Law 1988). The plates were stained with iodine and autoradiographed in a PhosphorImager Storm 860 laser scanner (Amersham Biosciences, Uppsala, Sweden); alternatively, the lipid spots were scraped and eluted and the radioactivity associated with each spot was determined by scintillation counting.
In vivo phospholipids synthesis by the posterior midgut and secretion to the luminal contents $-{ }^{3} \mathrm{H}-\mathrm{palm}-$ itic acid $\left(0.1 \mu \mathrm{L}, 10^{6} \mathrm{cpm}\right.$; specific activity: $57 \mathrm{mCi} /$ mmol) (Perkin Elmer, Waltham, Massachusetts, USA) was injected into adult female haemocoels 10 days after a blood meal using a PLI-100 microinjector (Harvard Apparatus, Holliston, Massachusetts, USA). After 2 $\mathrm{h}$, females were dissected, the luminal contents of the posterior midgut collected and subjected to lipid extraction and the phospholipids synthesised were analysed by TLC. The radioactivity associated with each phospholipid spot was determined by scintillation counting.

Purification of the R. prolixus PMM - An extract of the $R$. prolixus PMM and its discontinuous sucrose gradient fractions were obtained as described by Ferreira et al. (1988). Briefly, membrane fractions for sucrose gradient ultracentrifugation were prepared according to the following methods. One hour after the ${ }^{3} \mathrm{H}$-palmitic acid injection, posterior midgut homogenates were diluted five-fold with water and centrifuged at $25,000 \mathrm{~g}$ for $30 \mathrm{~min}$. The pellets were resuspended in water and after three freeze-thaw cycles, they were again centrifuged at $25,000 \mathrm{~g}$ for $30 \mathrm{~min}$. The pellets were resuspended in $0.2 \mathrm{~mL}$ and applied to the top of 4.6- $\mathrm{mL}$ linear gradients of sucrose $(4.5-45 \%, \mathrm{w} / \mathrm{v})$ prepared in 50 $\mathrm{mM}$ sodium acetate buffer $(\mathrm{pH} 5.5)$ containing $1 \mathrm{mg} / \mathrm{mL}$ bovine serum albumin (BSA). Gradient-centrifugations were performed at $96,000 \mathrm{~g}$ for $15 \mathrm{~h}$ at $4^{\circ} \mathrm{C}$. Fractions $(0.2 \mathrm{~mL})$ were collected, starting close to the bottom of the tubes (approximately $0.1 \mathrm{~mL}$ of the gradients were left behind to avoid collecting the pellet formed during ultracentrifugation). The activity of the marker enzyme $\alpha$-glucosidase (Ferreira et al. 1988) and the refractive index were determined for each fraction.

Lp purification - Lp was purified from the haemolymph by ultracentrifugation in a $\mathrm{KBr}$ gradient, as previously described (Gondim et al. 1989). Briefly, 5 $\mathrm{mL}$ of haemolymph were collected in the presence of a protease inhibitor cocktail (ethylenediamine tetraacetic acid and P8340, Sigma-Aldrich, St. Louis, Missouri, USA) and centrifuged at $16,000 \mathrm{~g}$ for $5 \mathrm{~min}$ at $4^{\circ} \mathrm{C}$ to remove haemocytes. $\mathrm{KBr}$ was added to the supernatant to a final concentration of $0.4 \mathrm{~g} / \mathrm{mL}$ and this mixture was again centrifuged at $125,000 \mathrm{~g}$ in a Beckman 50.1 Ti rotor (Brea, California, USA) at $4^{\circ} \mathrm{C}$ for $20 \mathrm{~h}$. The gradient was then fractionated from the top to the bottom and the Lp fractions were pooled, extensively dialysed against phosphate buffered saline and stored under liquid nitrogen until use. The degree of purification was monitored by sodium dodecyl sulphate (SDS) polyacrylamide gel electrophoresis and the protein concentration was estimated according to the method of Lowry et al. (1951) using a micro bicinchoninic acid Kit (Pierce, Rockford, Illinois, USA) in the presence of $0.3 \%(\mathrm{w} / \mathrm{v})$ SDS. BSA was used as a standard.

Statistical analysis - All data are presented as means \pm standard deviation of the mean. The means were determined from three independent assays. Standard error bars are not shown when the error range is smaller than 
the symbol size. Statistical comparisons of the means were evaluated using Student's $t$ test (unpaired) and a one-way analysis of variance followed by Dunnett tests (paired). A $\mathrm{p}$ value $\leq 0.05$ was considered significant.

\section{RESULTS}

When ${ }^{3} \mathrm{H}$-palmitic acid was added to the luminal side of posterior midguts and incubated in culture medium at $28^{\circ} \mathrm{C}$ for $1 \mathrm{~h}$, a significant amount of FAs was esterified. Fig. 1 shows the distribution of the radioactivity among the lipids of the posterior midgut $1 \mathrm{~h}$ after the incubation with ${ }^{3} \mathrm{H}$-palmitic acid. The lipids were analysed by TLC followed by autoradiography in the PhosphorImager. Phosphatidylethanolamine (PE), phosphatidylcholine (PC) and phosphatidylserine (PS) were the major phospholipids identified (Fig. 1), whereas DG and TG were the major NL identified (Fig. 1). The amounts of radioactivity accumulated in the phospholipids and the NL corresponded to approximately $80 \%$ and $20 \%$, respectively (Table I).

The rate of ${ }^{3} \mathrm{H}$-palmitic acid incorporation into the posterior midgut varied during the days following feeding. We observed that the capacity of the posterior midgut to absorb FAs from the luminal contents was higher in the first day after feeding (Fig. 2).

The time-course of ${ }^{3} \mathrm{H}$-palmitic acid esterification to DG and TG by the posterior midgut in the presence or absence of $\mathrm{Lp}$ in the incubation medium is shown in Fig. 3. The addition of $\mathrm{Lp}$ into the culture medium reduced the amount of ${ }^{3} \mathrm{H}-\mathrm{DG}$ s synthesised by the tissue and

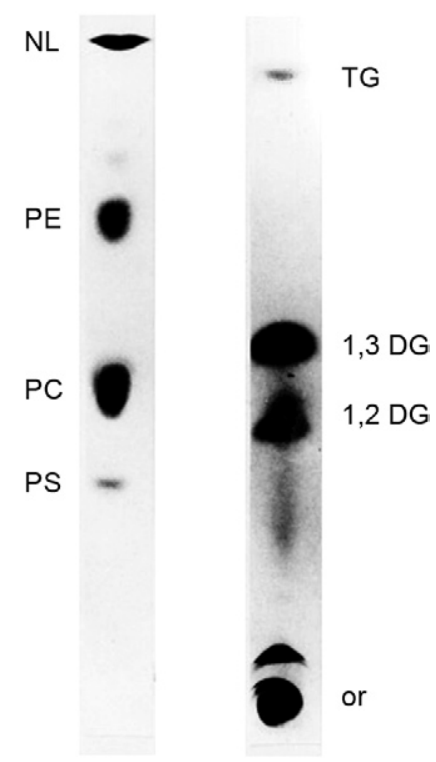

Fig. 1: neutral lipids (NL) and phospholipids the posterior midgut after contact with ${ }^{3} \mathrm{H}$-palmitic acid. The posterior midguts of adult females were dissected on the 10th day after blood feeding and ${ }^{3} \mathrm{H}$-palmitic acid was added at the luminal side and incubated in culture medium. Thirty minutes later, the midgut was washed and subjected to lipid extraction. The total lipids were analysed by thin-layer chromatography and autoradiographed in a PhosphorImager. DG: diacylglycerols; or: origin; PA: phosphatidic acid; PC: phosphatidylcholine; PE: phosphatidylethanolamine; PS: phosphatidylserine; TG: triacylglycerol. established a new steady state level (Fig. 3A). Similar results were obtained for the synthesis of ${ }^{3} \mathrm{H}-\mathrm{TG}$ in the posterior midgut. The addition of $\mathrm{Lp}$ in the culture medium reduced the amount of TG that was accumulated by the tissue (Fig. 3B).

In the 10th day after blood feeding, the ${ }^{3} \mathrm{H}-\mathrm{DG}$ that was synthesised by the posterior midgut was readily transferred to Lp, but the ${ }^{3} \mathrm{H}$-phospholipids remained in the tissue. The time-course of ${ }^{3} \mathrm{H}-\mathrm{DG}$ transfer is shown in Fig. 4. In the absence of Lp, a negligible amount of radioactivity was secreted into the medium. Despite the physiological state of the organ, a significant amount of the phospholip-

TABLE I

Total lipids synthesised by posterior midgut after contact with ${ }^{3} \mathrm{H}$-palmitic acid at the luminal side

\begin{tabular}{lcc}
\hline & cpm/midgut & $(\%)$ \\
\hline TG & $450 \pm 98$ & 3 \\
DG & $2,200 \pm 456$ & 16 \\
PC & $6,870 \pm 295$ & 49 \\
PE & $4,390 \pm 372$ & 32 \\
\hline Total & $13,910 \pm 2,212$ & 100
\end{tabular}

the posterior midguts of adult females were dissected on the 10th day after blood feeding and ${ }^{3} \mathrm{H}$-palmitic acid was added at luminal side and incubated in culture medium in the presence of lipophorin. Thirty minutes later, the posterior midgut was washed and subjected to a lipid extraction and the lipids were analysed by thin-layer chromatography. The spots were removed and eluted and the radioactivity associated with each lipid was estimated by scintillation counting. cpm: counts per minute; DG: diacylglycerols; PC: phosphatidylcholine; PE: phosphatidylethanolamine; TG: triacylglycerol.

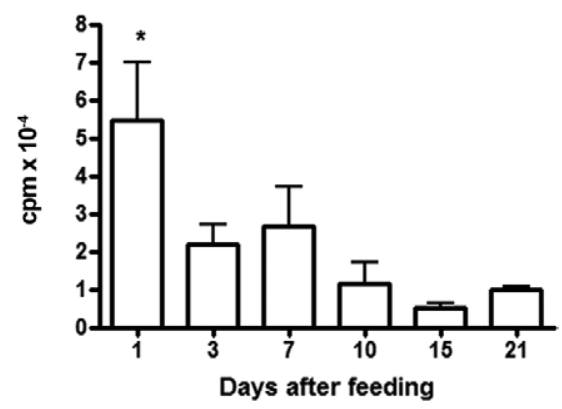

Fig. 2: ${ }^{3} \mathrm{H}$-palmitic acid incorporation by the posterior midgut in the days following feeding. Adult females were fed with non-radioactive blood. On different days after feeding, the posterior midguts of adult females were dissected and ${ }^{3} \mathrm{H}$-palmitic acid was added at the luminal side and incubated in the culture medium. One hour later, the midgut was dissected and homogenised and the radioactivity of the extracted lipids was determined by scintillation counting. The vertical bars represent the standard error for three determinations. Asterisk means the statistical analyses $\mathrm{p}<0.01$. cpm: counts per minute. 
ids that were synthesised by the posterior midgut (Table I) was not readily transferred to the culture medium associated Lp in the 10th day after feeding (Fig. 4).

We have previously demonstrated that the midgut of $R$. prolixus is able to take up FAs from the haemolymph (Atella et al. 2000). In vivo experiments were performed to understand whether the FAs derived from the haemolymph could be used by the midgut for phospholipid synthesis and secretion. ${ }^{3} \mathrm{H}$-palmitic acid was injected into the haemocoel and the phospholipids that were synthesised by the posterior midgut and secreted into luminal content were analysed by TLC. The radioactivity associated with each phospholipid spot was determined by scintillation counting. PC, PE, PS and phosphatidic acid (PA) were the major radioactive phospholipids identified (Table II, 1st column).

Ex vivo experiments were performed in parallel to compare the capacity of the luminal side of the posterior midgut to incorporate FAs and to synthesise phospho-

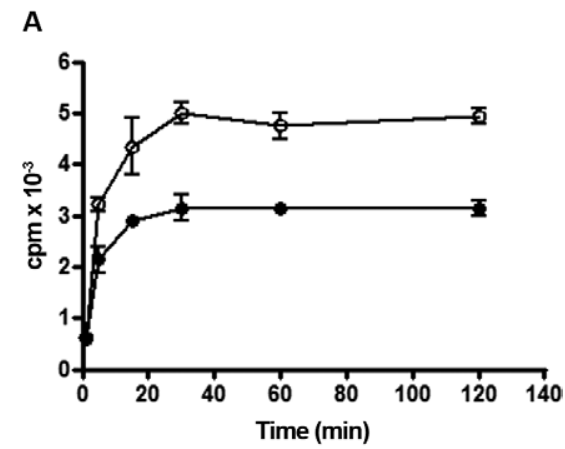

B

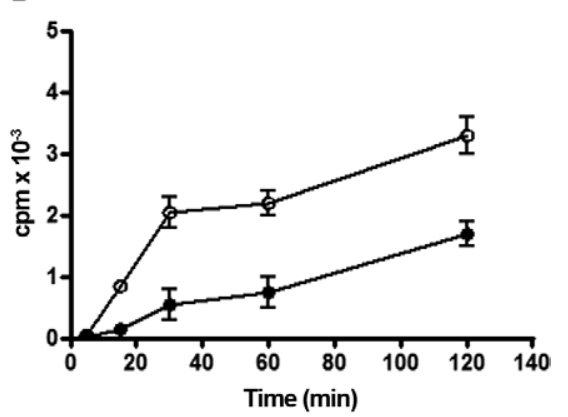

Fig. 3: time-course of ${ }^{3} \mathrm{H}$-palmitic acid esterification to diacylglycerol and triacylglycerol by the posterior midgut in the presence or absence of lipophorin (Lp). The posterior midguts of adult females were dissected on the 10th day after blood feeding and ${ }^{3} \mathrm{H}$-palmitic acid was added at the luminal side of the organ and incubated in culture medium at $28^{\circ} \mathrm{C}$ in the presence or absence of $\mathrm{Lp}$. At different times, the posterior midguts were removed from the culture medium, washed, homogenised and subjected to a lipid extraction and thin-layer chromatography. The lipid spots were scraped and the radioactivity associated with each spot was estimated by scintillation counting. Other experimental conditions are described in the Materials and Methods section. The vertical bars represent the standard error for four determinations. A: diacylglycerol from the midgut in the presence $(\bullet)$ or the absence (o) of Lp in the incubation medium; B: triacylglycerol from the midgut in the presence $(\bullet)$ or the absence ( $($ ) of $\mathrm{Lp}$ in the incubation medium. cpm: counts per minute. lipids. ${ }^{3} \mathrm{H}$-palmitic acid was injected into the luminal contents and incubated in culture medium. One hour later, the luminal contents were collected and subjected to lipid extraction and subsequent TLC. The radioactivity associated with each phospholipid spot was determined by scintillation counting. We observed radioactive PC, PE, PS and PA present in the luminal contents (Table II, 2nd column).

To investigate whether phospholipids found in the luminal contents were organised in PMMs, radioactive luminal contents from in vivo experiments (Table II, 1st column) were collected and PMMs were purified in a sucrose gradient. The phospholipids from purified PMMs were analysed by TLC. The radioactivity associated with each phospholipid spot was determined by scintillation counting. PE, PC and PS were the major phospholipids associated with the $R$. prolixus PMMs (Table III).

\section{DISCUSSION}

We have previously shown that the posterior midgut of $R$. prolixus is able to take up radioactive FAs from Lp preferentially on the 10th day after a blood meal (Atella et al. 1995). The synthesised NL were delivered as DG to Lp, but most of the phospholipids remained in the organ. In this study, we investigated the capacity of the posterior midgut to absorb radioactive FAs from the luminal side and to incorporate them into PMMs. When ${ }^{3} \mathrm{H}$-palmitic acid was added to the luminal side of the posterior midgut, it was readily absorbed and used to synthesise both phospholipids and NL (Fig. 1, Table I). In contrast to the fat bodies of L. migratoria and Manduca sexta (Soulages \& Wells 1994, Van der Horst et al. 2001), in which the majority of radioactive FAs were esterified as TG, in the $R$. prolixus midgut, $80 \%$ of the ${ }^{3} \mathrm{H}$-palmitic acid was used to synthesise phospholipids and only $20 \%$ was used to synthesise NL (Table I). These values are different from those obtained when ${ }^{3} \mathrm{H}$-palmitic acid was injected

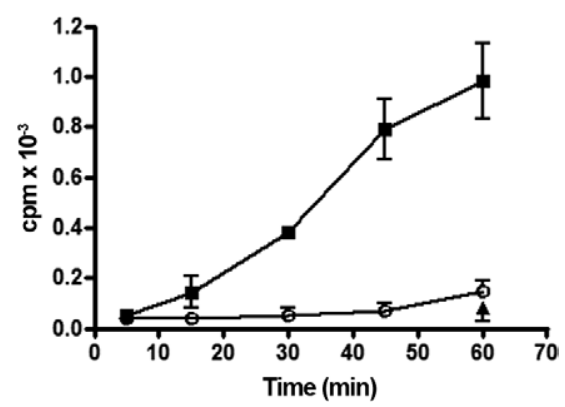

Fig. 4: time-course of ${ }^{3} \mathrm{H}$-diacylglycerol release to the medium associated with lipophorin (Lp) particles following the addition of ${ }^{3} \mathrm{H}$-palmitic acid at the luminal side. The posterior midguts of adult females fed 10 days earlier were dissected. ${ }^{3} \mathrm{H}$-palmitic acid was added at the luminal side and the organ was incubated in the presence of Lp. At different times, the incubation medium was collected subjected to a lipid extraction followed by thin-layer chromatography. ${ }^{3} \mathrm{H}$-diacylglycerol (घ), ${ }^{3} \mathrm{H}$-phospholipids (o) and total ${ }^{3} \mathrm{H}$-lipids in the absence of Lp ( $\boldsymbol{\Delta})$ were detected and the radioactivity was measured by scintillation counting. The vertical bars represent the error for four determinations. cpm: counts per minute. 
into the haemocoel of Rhodnius (Atella et al. 2000). In the latter case, $60 \%$ of ${ }^{3} \mathrm{H}$-palmitic acid was associated with phospholipids and $40 \%$ with NL. These differences can possibly be explained by the absence or presence of $\mathrm{Lp}$ in the experiments. Lp was present after injection of ${ }^{3} \mathrm{H}$-palmitic acid in the haemocoel, whereas Lp was absent in the incubation medium when ${ }^{3} \mathrm{H}$-palmitic acid was added at the luminal side of the posterior midgut. The removal of DG from the posterior midgut by Lp may have changed the distribution of lipids among different classes. In our case, the lack of Lp may have favoured phospholipid accumulation. Similar results were observed with respect to NL.

The addition of $L p$ to the incubation medium reduced the steady state levels of DG formation and the rate of TG accumulation, suggesting that TG is an end product and DG is an intermediary product (Fig. 3A, B). The major lipid released in the presence of Lp was DG and only a small amount of this lipid was detected in the medium when Lp was absent (Fig. 4). Phospholipids were poorly released, even in the presence of $\mathrm{Lp}$, which is consistent with the previous observations of Atella et al. (2000). The capacity of the posterior midgut to esterify ${ }^{3} \mathrm{H}$-palmitic acid after feeding reflects the physiological state of this tissue (Fig. 2). Soon after a blood meal, when the ingested blood starts to be digested, the posterior midgut is able to absorb the FAs to supply the lipid demand to other tissues, such as fat bodies and ovaries. Coelho et al. (1997) observed that soon after a blood meal, the midgut is able to transfer lipids to Lp circulating in the haemolymph. This transfer is associated with a decrease in particle density, indicating that the lipid/ protein ratio of the particles has changed towards lipids.

Regardless of whether FAs were added to the luminal or haemolymphatic sides, the major phospholipids syn-

\section{TABLE II}

${ }^{3} \mathrm{H}$-phospholipids present in the luminal content following injection of ${ }^{3} \mathrm{H}$-palmitic acid into the haemocoel or lumen

\begin{tabular}{lcc}
\hline & $\begin{array}{c}\text { Haemocoel } \\
(\%)\end{array}$ & $\begin{array}{c}\text { Lumen } \\
(\%)\end{array}$ \\
\hline PE & 53 & 49 \\
PC & 27 & 30 \\
PS & 12 & 13 \\
PA & 8 & 8 \\
\hline Total & 100 & 100 \\
\hline
\end{tabular}

${ }^{3} \mathrm{H}$-palmitic acid was injected into the haemocoel or lumen of adult females that had been fed with non-radioactive rabbit blood 10 days earlier. After different periods of time, the luminal content were collected, subjected to lipid extraction and analysed by thin-layer chromatography. The phospholipid spots were removed and eluted and the radioactivity associated with each different phospholipid was measured by scintillation counting. PA: phosphatidic acid; PC: phosphatidylcholine; PE: phosphatidylethanolamine; PS: phosphatidylserine. thesised were PE and PC, but PS and PA were also found in smaller amounts (Table II). The presence of PC and PA in the midgut is interesting because these phospholipids are substrates of phospholipase $\mathrm{A}_{2}$, which ultimately generates bioactive lysophospholipids, such as lysophosphatidylcholine (LPC) and lysophosphatidic acid.

In $R$. prolixus, our group has shown that salivary LPC is able to manipulate several signalling pathways within host cells, such as those related to the inhibition of platelet aggregation and nitric oxide production (Golodne et al. 2003). The overall outcome of such effects is the enhancement of T. cruzi transmission (Mesquita et al. 2008).

Another interesting aspect of our study is that regardless of the pathway for ${ }^{3} \mathrm{H}$-palmitic acid administration, the posterior midgut primarily absorbed and produced phospholipids that were excreted together with the luminal contents. It has previously been demonstrated that the gut of $R$. prolixus contains a large amount of extracellular membranes called PMMs that are progressively synthesised and released into the lumen (Terra 1988). Indeed, after injection of ${ }^{3} \mathrm{H}$-palmitic acid into the $R$. prolixus haemocoel, radioactive phospholipids were observed to be associated with purified PMMs (Table III). This result demonstrates that the posterior midgut is synthesising phospholipids, which are used primarily for the production of PMMs. Previous studies have shown that after the blood meal, PMMs acts as adhesion sites for $T$. cruzi epimastigotes. These studies suggest that glycoproteins from PMMs are important for the adhesion of the parasite to the posterior midgut cells. This mechanism appears to be essential for the establishment of the parasite in the insect vector (Garcia \& Azambuja 2004, Alves et al. 2007).

\section{TABLE III}

${ }^{3} \mathrm{H}$-phospholipids present in the purified perimicrovillar membranes (PMMs) following the injection of ${ }^{3} \mathrm{H}$-palmitic acid in the luminal content

\begin{tabular}{lcc}
\hline & cpm/midgut & $(\%)$ \\
\hline PE & $831 \pm 29$ & 51 \\
PC & $522 \pm 46$ & 32 \\
PS & $228 \pm 22$ & 14 \\
ND & $49 \pm 4$ & 3 \\
\hline Total & $1,630 \pm 97$ & 100
\end{tabular}

the posterior midguts of adult females that had been fed nonradioactive rabbit blood 10 days earlier were dissected and ${ }^{3} \mathrm{H}-$ palmitic acid was added into the luminal content and incubated in the culture medium. One hour later, the luminal content was collected and the PMMs were purified in a sucrose gradient and subjected to a lipid extraction. The phospholipids were then analysed by thin-layer chromatography. The spots were removed and eluted and the radioactivity associated with each spot was measured by scintillation counting. ND: not determinate; PC: phosphatidylcholine; PE: phosphatidylethanolamine; PS: phosphatidylserine. 
In insects, the use of micelle lysophospholipids is one of the strategies developed to facilitate lipid solubilisation during digestion, as insects do not synthesise bile salts (Turunen \& Kastari 1979, de Veau \& Schultz 1992, Canavoso et al. 2001). The presence of PC and LPC in the midgut of $R$. prolixus suggests that these molecules in this microenvironment can also work as surfactants, allowing the bioavailability of dietary lipids.

Future research should be conducted to determine the role of bioactive phospholipids in the midgut biology of insect vectors. Additionally, more studies are required to further understand the role of midgut lipids in $T$. cruzi infection.

In conclusion, this study shows that on the 10th day after a blood meal, the posterior midgut of $R$. prolixus used the incorporated FAs for the synthesis of different classes of lipids: TGs for storage, DG for exportation through Lp and phospholipids that were organised into PMMs and released together with the insect luminal contents.

\section{ACKNOWLEDGEMENTS}

To the memory of Alexandre A Peixoto, to Mileane S Busch, Lilian Soares da Cunha Gomes and Heloisa Coelho, for technical assistance, and to José de S Lima Jr and Gustavo Tavares Ali, for $R$. prolixus care.

\section{REFERENCES}

Alves CR, Albuquerque-Cunha JM, Mello CB, Garcia ES, Nogueira NF, Bourguingnon SC, de Souza W, Azambuja P, Gonzalez MS 2007. Trypanosoma cruzi: attachment to perimicrovillar membrane glycoproteins of Rhodnius prolixus. Exp Parasitol 116: 44-52.

Araújo CA, Mello CB, Jansen AM 2002. Trypanosoma cruzi I and Trypanosoma cruzi II: recognition of sugar structures by Arachis hypogaea (peanut agglutinin) lectin. J Parasitol 88: 582-586.

Atella GC, Arruda MA, Gondim KC, Masuda H 2000. Fatty acids incorporation by Rhodnius prolixus midgut. Arch Insect Biochem Physiol 43: 99-107.

Atella GC, Gondim KC, Masuda H 1995. Loading of Lp particles with phospholipids at the midgut of Rhodnius prolixus. Arch Insect Biochem Physiol 30: 337-350.

Baerwald RJ, Delcarpio JB 1983. Double membrane-bounded intestinal microvilli in Oncopeltus fasciatus. Cell Tissue Res 232: 593-600.

Bauerfeind R, Komnick H 1992. Lipid-loading and unloading of Lp in the midgut epithelium of dragonfly larvae (Aeshna cyanea) A biochemical and immunocytochemical study. J Insect Physiol 38: $147-160$.

Beenakkers AM, Van der Horst DJ, Van Marrewijk WJ 1985. Insect lipids and lipoproteins and their role in physiological processes. Prog Lipid Res 24: 19-67.

Billingsley PF, Downe AE 1986. The surface morphology of the midgut cells of Rhodnius prolixus Stål (Hemiptera: Reduviidae) during blood digestion. Acta Trop 43: 355-366.

Bligh EG, Dyer WJ 1959. A rapid method of total lipid extraction and purification. Can J Biochem Physiol 37: 911-917.

Bourguignon SC, de Souza W, Souto-Padrón T 1998. Localization of lectin-binding sites on the surface of Trypanosoma cruzi grown in chemically defined conditions. Histochem Cell Biol 110: 527-534.

Burgos MH, Gutiérrez LS 1976. The intestine of Triatoma infestans. I. Cytology of the midgut. J Ultrastruct Res 57: 1-9.

Canavoso LE, Jouni ZE, Karnas KJ, Pennington JE, Wells MA 2001. Fat metabolism in insects. Annu Rev Nutr 21: 23-46.
Coelho HSL, Atella GC, Moreira MF, Gondim KC, Masuda H 1997. Lp density variations during oogenesis of Rhodnius prolixus. Arch Insect Biochem Physiol 35: 301-313.

de Veau IEJ, Schultz JC 1992. Reassessment of interaction between gut detergents and tannins in lepidoptera and significance for gypsy moth larvae. J Chem Ecol 18: 1437-1453.

Ferreira C, Ribeiro AF, Garcia ES, Terra RW 1988. Digestive enzymes trapped between and associated with the double plasma membranes of Rhodnius prolixus posterior midgut cells. Insect Biochem 18: 521-530.

Garcia ES, Azambuja P 2004. Lignoids in insects: chemical probes for the study of ecdysis, excretion and Trypanosoma cruzi-triatomine interaction. Toxicon 44: 431-440.

Golodne DM, Monteiro RQ, Graça-Souza AV, Silva-Neto MA, Atella GC 2003. Lysophosphatidylcholine acts as an anti-hemostatic molecule in the saliva of the blood-sucking bug Rhodnius prolixus. J Biol Chem 278: 27766-27771.

Gondim KC, Oliveira PL, Coelho HSL, Masuda H 1989. Lp from Rhodnius prolixus: purification and partial characterization. Insect Biochem 19: 153-161.

Gonzalez MS, Nogueira NF, Mello CB, de Souza W, Schaub GA, Azambuja P, Garcia ES 1999. Influence of brain and azadirachtin on Trypanosoma cruzi development in the vector, Rhodnius prolixus. Exp Parasitol 92: 100-108.

Grillo LA, Majerowicz D, Gondim KC 2007. Lipid metabolism in Rhodnius prolixus (Hemiptera: Reduviidae): role of a midgut triacylglycerol-lipase. Insect Biochem Mol Biol 37: 579-588.

Horwitz J, Perlman RL 1987. Measurement of inositol phospholipid metabolism in PC12 pheochromocytoma cells. Methods Enzymol 141: 169-175.

Isola EL, Lammel EM, González Cappa SM 1986. Trypanosoma cru$z i$ : differentiation after interaction of epimastigotes and Triatoma infestans intestinal homogenate. Exp Parasitol 62: 329-335.

Kawooya JK, Law JH 1988. Role of Lp in lipid transport to the insect egg. J Biol Chem 263: 8748-8753.

Kollien AH, Schmidt J, Schaub GA 1998. Modes of association of Trypanosoma cruzi with the intestinal tract of the vector Triatoma infestans. Acta Trop 70: 127-141.

Lane NJ, Harrison JB 1979. An unusual cell surface modification: a double plasma membrane. J Cell Sci 39: 355-372.

Lowry OH, Rosenbrough NJ, Farr AL, Randall RJ 1951. Protein measurement with the folin phenol reagent. J Biol Chem 193: 265-275.

Marshall AT, Cheung WW 1970. Ultrastructure and cytochemistry of an extensive plexiform surface coat on the midgut cells of a fulgorid insect. J Ultrastruct Res 33: 161-172.

Mello CB, Azambuja P, Garcia ES, Ratcliffe NA 1996. Differential in vitro and in vivo behavior of three strains of Trypanosoma cruzi in the gut and hemolymph of Rhodnius prolixus. Exp Parasitol 82: 112-121.

Mesquita RD, Carneiro AB, Bafica A, Gazos-Lopes F, Takiya C, Souto-Padron T, Vieira DP, Ferreira-Pereira A, Almeida I, Figueiredo RT, Bozza MT, Graça-Souza A, Lopes AH, Atella GC, Silva-Neto MA 2008. Trypanosoma cruzi infection is enhanced by vector saliva through immunosuppressant mechanisms mediated by lysophosphatidylcholine. Infect Immun 76: 5543-5552.

Mury FB, da Silva JR, Ferreira LS, dos Santos Ferreira B, de SouzaFilho GA, de Souza-Neto JA, Ribolla PE, Silva CP, do Nascimento VV, Machado OL, Berbert-Molina MA, Dansa-Petretski M 2010. Alpha-glucosidase promotes hemozoin formation in a bloodsucking bug: an evolutionary history. PLOS ONE 4: e6966. 
Nogueira NF, Gonzales M, Garcia EM, de Souza W 1997. Effect of azadirachtin A on the fine structure of the midgut of Rhodnius prolixus. J Invertebr Pathol 69: 58-63.

Pereira NM, Königk E 1981. A nucleotidase from Leishmania tropica promastigotes: partial purification and properties. Tropenmed Parasitol 32: 209-214.

Ratcliffe NA, Nigam Y, Mello CB, Garcia ES, Azambuja P 1996. Trypanosoma cruzi and erythrocyte agglutinins: a comparative study of occurrence and properties in the gut and hemolymph of Rhodnius prolixus. Exp Parasitol 83: 83-93.

Reger JF 1971. An unusual membrane organization observed during spermiogenesis in the mite Caloglyphus anomalus. J Ultrastruct Res 36: 732-742.

Silva CP, Ribeiro AF, Terra WR 1996. Enzyme markers and isolation of the microvillar and perimicrovillar membranes of Dysdercus peruvianus (Hemiptera: Pyrrhocoridae) midgut cells. Insect Biochem Mol Biol 26: 1011-1018.

Silva JR, Mury FB, Oliveira MF, Oliveira PL, Silva CP, Dansa-Petretski M 2007. Perimicrovillar membranes promote hemozoin formation into Rhodnius prolixus midgut. Insect Biochem Mol Biol 37: 523-531.
Soulages JL, Wells MA 1994. Lp: the structure of an insect lipoprotein and its role in lipid transport in insects. Adv Protein Chem 45: 371-415.

Terra WR 1988. Physiology and biochemistry of insect digestion: an evolutionary perspective. Braz J Med Biol Res 21: 675-734.

Terra WR, Costa RH, Ferreira C 2006. Plasma membranes from insect midgut cells. An Acad Bras Cienc 78: 255-269.

Turunen S 1975. Absorption and transport of dietary lipid in Pieris brassicae. J insect Physiol 21: 1521-1529.

Turunen S, Kastari T 1979. Digestion and absorption of lecithin in larvae of the cabbage butterfly, Pieris brassicae. Comp Biochem Physiol 62A: 933-937.

Van der Horst DJ, Van Marrewijk WJ, Diederen JH 2001. Adipokinetic hormones of insect: release, signal transduction and responses. Int Rev Cytol 211: 179-240.

Weintraub H, Tietz A 1973. Triglyceride digestion and absorption in the locust. Locusta migratoria. Biochim Biophys Acta 306: $31-41$.

Wigglesworth VB 1928. Digestion in the cockroach. Biochem J 22: 150-161. 